\title{
COMPLICATIONS OF THE INDWELLING URETHRAL CATHETER
}

\author{
A. G. HARDY, M.B.E., B.M., F.R.C.S.
}

THE management of the paralysed bladder is only one important part of the treatment of traumatic paraplegia. All aspects must have due consideration at any one time and each is as much dependent on the whole as the whole is dependent on each integral part. Sores, sepsis, muscle spams, prolonged recumbency and immobility can all produce profound functional and structural changes in the urinary tract and a true appreciation of the significance of the indwelling urethral catheter can only be made if the cases under consideration have had a comparable programme of treatment in all other respects. Ideally the cases should also be free from other complications in the initial instance.

TABLE I

Catheter Periods

\begin{tabular}{|l|l|c|c|c|}
\hline Bony Level & $\begin{array}{l}\text { Neurological } \\
\text { state }\end{array}$ & Cases & \multicolumn{2}{|c|}{ Catheter periods } \\
\hline Cervical & $\begin{array}{l}\text { complete } \\
\text { partial }\end{array}$ & $\begin{array}{r}\text { Actual } \\
\text { I05 }\end{array}$ & Average \\
\hline Dorsal . & $\begin{array}{l}\text { I to } 50 \\
\text { I to } 35\end{array}$ & 24 \\
\hline complete & 69 & 3 to 56 & I0 \\
\hline portial & 23 & 4 to 25 & 9 \\
\hline Lumbar & $\begin{array}{l}\text { complete } \\
\text { partial }\end{array}$ & 92 & 8 to 46 & I7 \\
\hline
\end{tabular}

For the purpose of this paper an analysis is made of a group of 400 new cases in a Io-year period between I956 and 1965. All these cases were admitted within a day or days from the time of injury and all had the same regime of general management as well as the same method of bladder treatment. There were I39 cases of cervical spinal cord injury, 92 cases of dorsal cord injury, I34 cases at the dorso lumbar junction and 35 in the lumbar region. These cases were further subdivided into two groups from the neurological aspect and each anatomical region was classified with having a lesion which was either complete or incomplete. There was no difficulty in identifying a complete lesion but the incomplete lesions showed considerable variation in degrees of incompleteness.

Catheter Periods. The catheter is a foreign body and the duration of the period of need for catheter drainage is obviously important, not only because of the 
length of time that a foreign body is present in the lower urinary tract but because of the number of instrumentations which are necessary during that period. The first consideration was to look at the catheter periods for the different groups of cases. These are shown in Table I and as one might expect the cases with complete lesions required approximately twice as long a period of drainage as the cases with incomplete lesions. A study of the events that occurred in all these cases forms a presentation of the experiences with catheter drainage of the paralysed bladder and the first observations are on the catheter itself.

Catheter Defects. The main experience has been with the use of the self retaining balloon type catheter more generally referred to as the Foley. The sizes used have been from 16 to 22 French gauge and the balloon capacity has been labelled $5 \mathrm{cc}$.

In earlier years these catheters were not always satisfactory because of a number of defects in design and manufacture. The defects had a very definite nuisance value in so far as they caused interruption in the continuous and adequate flow of urine from the bladder. They also caused a need for additional instrumentation which would not otherwise have been necessary. A failure of drainage could result in retention of urine with distension of the bladder and potential flare up of urinary tract infection.

The defects included:

I. Insidious deflation of the balloon.

2. Occlusion of the lumen of the catheter by pressure within the balloon.

3. Occlusion of the lumen of the catheter by the distortion of a balloon blown up eccentrically.

4. Failure of the balloon to deflate when required.

An awareness of these defects and prompt action to alleviate the effects of them was a necessary part of the bladder drainage programme.

Insidious deflation of the balloon added to the risks of the method in so far as it meant that the catheter came out of the bladder and over distension occurred if the incident was not discovered. In addition there were the added risks because of the need for another instrumentation.

Occlusion of the lumen of the catheter by pressure within the balloon or by distortion of the balloon because it blew up eccentrically gave rise to an impairment of drainage which could be recognised by observing the flow of urine before and after the balloon was blown up. This was actually a common feature in earlier years and much more so than it is now. It merely necessitated a prompt change of catheter for a better one.

Failure of the balloon to deflate when required occurred from time to time. If the lumen of the catheter was not blocked there was no urgency of action and the side tube could be left open to see if the balloon fluid would drain away over a period. Failing this the normal practice was to take the case to the operating theatre and thread the catheter through a direct vision urethroscope and puncture the balloon with a long stilette.

Another method used was the introduction of a very small quantity of chloroform up the side tube. This dissolved the latex of the balloon within seconds and the catheter could be withdrawn immediately. In this method it is necessary to first check that the balloon will accept more fluid and secondly to introduce a little 
sterile paraffin into the bladder to minimise the irritant effects that the chloroform may produce on the mucous membrane of the bladder.

Bursting balloons by over distension has not been practised although it is known that this can and has been done. It is also known that this method has been responsible for fragments of rubber and in particular the rubber ring at the base of the balloon being left behind in the bladder. A calculus with a rubber core has been removed on Io occasions and one of them included the complete end of a catheter.

In more recent years the quality and mechanical efficiency of the self retaining balloon catheter have greatly improved and complications arising from faults in the catheter itself are much more rarely seen.

The more common use of polythene type tubing in the manufacture of catheters and drainage tubing has greatly facilitated the direct observation of urine drainage. With transparent tubing, faults in anv drainage system can be readily and promptly recognised.

Local Complications. Local complications of the indwelling urethral catheter arise as a result of the physical irritation of the catheter itself and/or the infections which occur as a result of its presence. Local complications are a risk on every occasion an instrument is passed up the urethra and into the bladder. Complications can be greatly minimised by care in the choice and size of the catheter and by meticulous attention to the details of asepsis in the techniques of the procedure. The three common sites of local physical irritation are at the external meatus, the peno-scrotal junction and the internal urethral orifice.

In the series under consideration the main urethral complications are listed in Table II. The first item requires further qualification as the 18 cases identified are

\section{TABLE II}

Urethral Complications I956-I965. 400 Cases

\begin{tabular}{|c|c|c|c|}
\hline Infection & • & & \\
\hline Abscess & & & \\
\hline Diverticulum & & & \\
\hline Fistula & & & \\
\hline Stricture & . & & \\
\hline
\end{tabular}

only those that had had a frankly purulent discharge lasting for more than 48 hours. In the earlier part of the Io year period cultures were taken from the urethra at the time of catheterisations and in almost every instance some pathogen was demonstrated. These have not been included as infections in the strictest sense of the word.

Periurethral abscesses developed on four occasions and all four ruptured into the urethral lumen and resulted in the diverticulae which have remained a permanent feature. There were no cases of fistula in any one of these early cases.

Urethral stricture occurred in association with a purulent urethritis and was undoubtedly due to a failure to observe that the catheter was too tight for too long a period. In the other cases of purulent urethritis a smaller size and type of catheter was needed until the local condition had been brought under control.

The comparatively low incidence of local infections and certainly the absence of any fistulas have been due to the fact that the catheters were selected with care. 
The catheter should be neither too big nor too small and of a size sufficient to allow free drainage of any mucus between it and the mucous membrane of the walls of the urethra.

Care was also taken to avoid traction on the cather as it lay in the penile urethra. This was achieved by taping the penis and catheter up on to the abdomen.

General Complications. Infections and Calculi. All but a very small number of the 400 cases had some infection at some time during the catheter period. The few that were successfully managed without any infection were those that had very short periods of catheter drainage and all of these were cases with incomplete neurological lesions.

The infections that are listed in Table III again require some qualification.

TABLE III

Infections and Calculi

\begin{tabular}{|c|c|c|c|c|}
\hline Bony level & $\begin{array}{c}\text { Neurological } \\
\text { state }\end{array}$ & Cases & Infections & Calculi \\
\hline Cervical & $\begin{array}{l}\text { complete } \\
\text { partial }\end{array}$ & $\begin{array}{r}34 \\
105\end{array}$ & $\begin{array}{l}12 \\
23\end{array}$ & $\begin{array}{l}7 \\
5\end{array}$ \\
\hline Dorsal . & $\begin{array}{l}\text { complete } \\
\text { partial }\end{array}$ & $\begin{array}{l}69 \\
23\end{array}$ & $\begin{array}{r}30 \\
4\end{array}$ & $\begin{array}{r}\mathrm{I} 2 \\
\mathrm{I}\end{array}$ \\
\hline Dorso lumbar & $\begin{array}{l}\text { complete } \\
\text { partial }\end{array}$ & $\begin{array}{l}92 \\
42\end{array}$ & $\begin{array}{r}23 \\
5\end{array}$ & $\begin{array}{r}18 \\
6\end{array}$ \\
\hline Lumbar & & 35 & 3 & 0 \\
\hline
\end{tabular}

These relate to those that were associated with the production of pus and a constitutional upset with associated clinical features. The incidence in these cases was greatest in cases with complete lesions than in incomplete lesions. However this may be related to the greater number of instrumentations rather than the fact that the neurological lesions were complete or incomplete. It is also interesting to note that the infections were greatest in some years or parts of years and this fact is further illustrated in a study of the incidence of infections of the epidydimis.

Calculi. The calculi referred to in Table III are those which were found during or at the end of a period of catheter drainage. They were all of the small flat type and clearly formed from phosphatic debris which had previously been encrusted on the Foley balloon. They were all easily crushed with a biopsy forceps and washed out through a suitable evacuator. The incidence of calculus formation has actually dropped quite considerably since our use of intermittent catheterisation in selected cases. It was also very considerably less in those cases who had managed to maintain a high fluid intake, although lack of infections was another important factor.

Infections of the Epidydimis. The incidence of infections of the epidydimis is shown in Table IV. Infections were much more common during the period of 
catheter drainage than at times when there was no drainage. Further observation on these figures shows the wide range of incidence over the years with a low of 7 per cent. to a high of 33 per cent. It is still not known exactly why this variation occurred, although in 1963 there was undoubtedly a very virulent infection running through the ward.

TABLE IV

Infections of the Epidydimis

\begin{tabular}{|c|c|c|c|c|c|c|c|c|c|c|}
\hline Year. & 1956 & I957 & I958 & 1959 & 1960 & I96I & 1962 & 1963 & I964 & 1965 \\
\hline New cases & 34 & 32 & 40 & 37 & 33 & 47 & 34 & 45 & 56 & 42 \\
\hline Catheter & 3 & 2 & 4 & 4 & 4 & 3 & 4 & IO & 6 & 2 \\
\hline No catheter & 0 & I & 2 & 3 & I & 2 & 3 & 5 & 5 & I \\
\hline Total & $\begin{array}{c}3 \\
9 \%\end{array}$ & $\begin{array}{r}3 \\
10 \%\end{array}$ & $\begin{array}{c}6 \\
15 \%\end{array}$ & $\begin{array}{c}7 \\
20 \%\end{array}$ & $\begin{array}{r}5 \\
15 \%\end{array}$ & $\begin{array}{c}5 \\
\text { II } \%\end{array}$ & $\begin{array}{c}7 \\
20 \%\end{array}$ & $\begin{array}{c}15 \\
33 \%\end{array}$ & $\begin{array}{c}\text { I I } \\
20 \%\end{array}$ & $\begin{array}{c}3 \\
7 \%\end{array}$ \\
\hline
\end{tabular}

Closer analysis of these cases of infection of the epidydimis with or without orchitis and abscess revealed a number of interesting facts. It is noted that they frequently followed a change of catheter or some investigation made with the use of a catheter. Thus incidents occurred following trial periods without the catheter, observations following the introduction of ice water, after cystograms and routine cystoscopy. Finally a very definite number of cases followed over distension resulting from a blocked catheter.

Multiple Complications. The complications were sometimes multiple in the same case and some cases had a series of complications in sequence. There are thus fewer cases involved than is apparent by first examination of the list of complications.

The complications were certainly not specifically related to the length of period of time of drainage by indwelling catheter, although the cases that had had the longest periods of catheter drainage had also had the greatest number of separate instrumentations of one sort and another. A critical period for the appearance of the urethral complications appeared to be in the early weeks and the incidence of infections of the epidydimis was more frequent in the eighth to twelfth week. This latter occurrence may be related to the fact that this particular period was a time of intensive observation on bladder activity in regard to reflex activity and evidence of voiding ability.

\section{DISCUSSION}

From these observations it would seem that infections make up the most significant part of the complications of the indwelling urethral catheter and that cases with infection of the epidydimis were the most troublesome. No prophylactic antibiotic or chemotherapeutic treatment was given but the usual range of medicinal 
therapy appropriate to the culture and sensitivity of the organism was used in active infections. It might be thought advantageous to perform some of the investigative procedures under cover of antibiotic therapy. This is not justified unless there have been previous disturbances as a result of the same procedure.

The technique of catheterisation included apparatus which had been prepacked and autoclaved. There was a separate pack for each procedure in each case. Chlorhexidine solution I : 2000 was used for cleaning the meatus and prepuce and I per cent. Chlorhexidine cream was instilled into the urethra before the passage of the catheter. The latter procedure was omitted on occasions when urine specimens were being taken for examination.

The catheters were changed each week and each catheter was attached to a closed drainage system comprising a polythene bag and drainage tube. Connections from the catheter to the bag were stored in Chlorhexidine solution. The collecting bags were changed twice daily and at times when a patient was up in a chair the end of the catheter and the end of the drainage tube were both covered with sterile caps. 